\title{
FRW Universe Models in Conformally Flat Spacetime Coordinates III: Universe models with positive spatial curvature
}

\author{
Øyvind Grøn* and Steinar Johannesen*
}

* Oslo University College, Faculty of Engineering, P.O.Box 4 St.Olavs Plass, N-0130 Oslo, Norway

\begin{abstract}
We deduce general expressions for the line element of universe models with positive spatial curvature described by conformally flat spacetime coordinates. Models with dust, radiation and vacuum energy are exhibited. Discussing the existence of particle horizons we show that there is continual annihilation of space, matter and energy in a dust and radiation dominated universe, and continual creation in a LIVE domined universe when conformal time is used in Friedmann-Robertson-Walker models with positive spatial curvature. A general procedure is given for finding coordinates to be used in Penrose diagrams. We also calculate the age and the redshift of some universe models using conformal time.
\end{abstract}

\section{Introduction.}

In order to complete the description of the FRW universe models in conformally flat spacetime (CFS) coordinates as given in part I and II of this series [1,2], it remains to describe the universe models with positive spatial curvature. From the general formalism presented in part I of this series, we find in section 2 the coordinate transformations to a CFS description of such universe models. Combining the transformations described in part II of this series with the inverse of the transformations in section 2, we obtain Penrose diagrams in section 3 . We also present some results concerning physical properties of FRW universe models as described in CFS coordinates, including the Hubble parameter, the CFS age of the universe, the recession velocity and the cosmic redshift. In this paper we shall refer to equations in reference [1] or [2] by writing I or II:(equation number).

\section{Universe models with positive spatial curvature}

\subsection{Conformal coordinates in positively curved universe models}

We shall now consider positively curved universe models. Then we can introduce conformal coordinates $(T, R)$ by choosing $a=0, b=0, c=1$ and $d=0$ in equation I:(33). This gives the generating function

$$
f(x)=\tan (x / 2) .
$$

The transformation I:(28) then takes the form $[3,4]$

$$
T=\frac{\sin \eta}{\cos \eta+\cos \chi} \quad, \quad R=\frac{\sin \chi}{\cos \eta+\cos \chi},
$$

transforming the triangle defined by $0<\chi<\pi$ and $0<\eta<\pi-\chi$ onto the first quadrant $R>0, T>0$, and the triangle defined by $0<\chi<\pi$ and $\pi+\chi<\eta<2 \pi$ onto the 
fourth quadrant $R>0, T<0$. We also have a conformal coordinate system $(\widetilde{R}, \widetilde{T})$ given by the same formulae (2), which maps the triangle defined by $0<\chi<\pi$ and $\pi-\chi<\eta<\pi+\chi$ onto the left half plane $\widetilde{R}<0$. Note that the negative sign of $\widetilde{R}$ can be removed by changing the coordinates $\theta$ and $\phi$ corresponding to a reflection through the origin in space. These domains are those needed in universe models with dust and radiation. We will later specify the domains needed for universe models with vacuum energy. The inverse transformation is

$$
\cot \eta=\frac{1-\left(T^{2}-R^{2}\right)}{2 T} \quad, \quad \cot \chi=\frac{1+\left(T^{2}-R^{2}\right)}{2 R} .
$$

The world lines of the cosmic reference particles, $\chi=\chi_{0}$, as given in the conformal system, are

$$
\left(R+a_{4}\right)^{2}-T^{2}=a_{4}^{2}+1
$$

where $a_{4}=\cot \chi_{0}$, describing hyperbolae with centra at $\left(-a_{4}, 0\right)$. The simultaneity curves of the cosmic space, $\eta=\eta_{0}$, are

$$
\left(T+b_{4}\right)^{2}-R^{2}=b_{4}^{2}+1,
$$

where $b_{4}=\cot \eta_{0}$, describing hyperbolae with centra at $\left(-b_{4}, 0\right)$. These curves are shown in Figure 1 in the first quadrant.

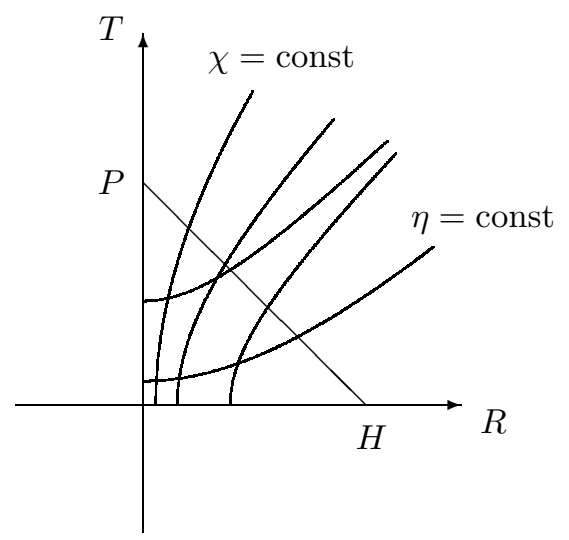

Figure 1. The first quadrant of the $(T, R)$-diagram for universe models with positive spatial curvature with reference to the conformal coordinate system defined in equation (2).

Note that all points on a simultaneity curve come arbitrary close to the $R$-axis in the limit $\eta_{0} \rightarrow 0$. Hence, in this coordinate system, the Big Bang occurred everywhere at the moment $T=0$. Therefore there is no continual creation of conformal space in this coordinate system. This is different from Big Bang as described with reference to a conformal coordinate system of type I in a negatively curved universe model where the Big Bang occurred along the light cone in Figure 3 of reference [2].

The line element now takes the conformally flat form [3 - 5]

$$
d s^{2}=\frac{4 a(\eta(T, R))^{2}}{4 T^{2}+\left[1-\left(T^{2}-R^{2}\right)\right]^{2}} d s_{M}^{2},
$$


where the scale factor depends upon the matter and energy contents of the universe. In a universe with dust and radiation the line element is

$$
d s^{2}=\left[\frac{2 \alpha\left\{\sqrt{4 T^{2}+\left[1-\left(T^{2}-R^{2}\right)\right]^{2}}-\left[1-\left(T^{2}-R^{2}\right)\right]\right\}+4 \beta T}{4 T^{2}+\left[1-\left(T^{2}-R^{2}\right)\right]^{2}}\right]^{2} d s_{M}^{2} .
$$

From equation I:(46) we obtain the following expression for the recession velocity

$$
V=\frac{2 T R}{1+T^{2}+R^{2}}=\frac{\sin \eta \sin \chi}{1+\cos \eta \cos \chi}
$$

Once more the initial recession velocity vanishes.

\subsection{Particle horizon in positively curved universe models with radiation and dust}

We shall here give a geometrical discussion of the particle horizon in universe models with positive spatial curvature with reference to the conformal coordinate system defined in equation (2).

In general, using cosmic coordinates, the particle horizon is given in equation I:(9). Let us see how this can be deduced by a geometrical consideration based upon Figure 1. In this figure $P$ is an observation event at the point of time $T_{0}$.

The horizon is the intersection of his backwards light cone with the space defined by $\eta=0$. This intersection is at the point $H$ in Figure 1, and from equation (2) it follows that the horizon is represented by the $R$-axis in the figure.

The conformal coordinates of the point $H$ are $T_{H}=0$ and $R_{H}=T_{0}$. Inserting this into equation (4) of a hyperbola $\chi=\chi_{H}$ gives

$$
\cot \chi_{H}=\frac{1-T_{0}^{2}}{2 T_{0}}
$$

We also need to find the $\eta$-coordinate of $P$. The conformal coordinates of $P$ are $T_{P}=T_{0}$ and $R_{P}=0$. Inserting this into equation (5) of a hyperbola $\eta=\eta_{P}$ gives

$$
\cot \eta_{P}=\frac{1-T_{0}^{2}}{2 T_{0}}
$$

Hence $\chi_{H}=\eta_{P}$ in accordance with equation I:(10).

In a positively curved universe with radiation and dust

$$
a=\alpha(1-\cos \eta)+\beta \sin \eta
$$

and

$$
t=\alpha(\eta-\sin \eta)+\beta(1-\cos \eta)
$$

where

$$
\alpha=\frac{\Omega_{m 0}}{2\left(\Omega_{0}-1\right)} \quad \text { and } \quad \beta=\sqrt{\frac{\Omega_{\gamma 0}}{\Omega_{0}-1}}
$$


For this universe model the relationship between the cosmic time and conformal time at $R=0$ is

$$
t=\alpha\left(\arcsin \frac{2 T}{1+T^{2}}-\frac{2 T}{1+T^{2}}\right)+\beta \frac{2 T^{2}}{1+T^{2}} .
$$

The scale factor $a(\eta)$ increases from zero at $\eta_{1}=0$ to a maximal value at

$$
\eta_{2}=\pi-\arctan \frac{\beta}{\alpha}
$$

and decreases to zero at $\eta_{3}=2 \eta_{2}$. In a radiation dominated universe $\alpha=0$ which gives $\eta_{3}=\pi$. In a dust dominated universe $\beta=0$ giving $\eta_{3}=2 \pi$.

In a closed universe the coordinate $\chi$ is defined in the interval $<0, \pi\rangle$ which means that the whole universe is covered except some points where the coordinates are not uniquely defined. Imagine an observer at $\chi=0$. At a point of time $\eta$ he can see objects at $\chi<\chi_{H}$ where $\chi_{H}=\eta$ is the radius of his particle horizon. When $\chi_{H}=\pi$ the whole universe is inside the horizon. Then the observer can see all of the universe. In a universe existing at $\eta>\pi$ there is no longer any particle horizon. In a universe with $k=1$ and radiation only the Big Crunch occurs at $\eta=\pi$, corresponding to $T=0$. The whole univese can only be seen at this final moment. In a matter dominated universe the Big Crunch occurs at $\eta=2 \pi$. Then the whole universe can be seen at the moment of maximum extension. At this moment the particle horizon vanishes.

Consider a free particle with $\chi=\chi_{0}$ in a universe with dust. When the parametric time approaches $\pi-\chi_{0}$, then $T \rightarrow \infty$ and $R \rightarrow \infty$. The conformal clocks go increasingly fast relative to the cosmic and the parametric clocks. The relative rate approaches infinity as $\eta \rightarrow \pi-\chi_{0}$. Hence the spacetime can not be covered by a single conformal coordinate system. We must use two coordinate systems $(T, R)$ and $(\widetilde{T}, \widetilde{R})$ covering the domains I and II in the $(\eta, \chi)$-plane as shown in Figure 2.
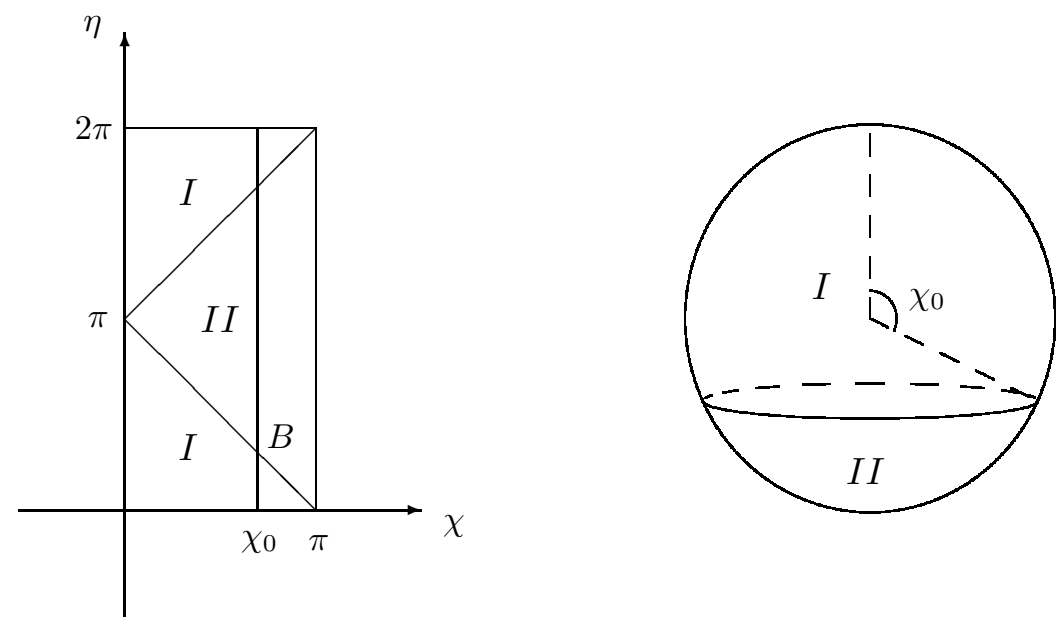

Figure 2. The domains of the conformal coordinate systems defined in equation (21) for dust dominated universe models. The right hand part of the figure shows the spherical 3-space of the closed universe model, suppressing the angular coordinate $\theta$. The coordinate $\chi$ has the value $\chi=0$ at the top of the figure which we call the North pole, and the value $\chi=\pi$ at the South pole. At $\chi=\chi_{0}$ the point $B$ in the left hand part of the figure corresponds to the circle between the regions I and II on the right hand part. 
The particle with $\chi=\chi_{0}$ enters the domain II at the parametric time $\pi-\chi_{0}$, and comes back to domain I at the time $\pi+\chi_{0}$. The motion of the particle in the conformal coordinate systems looks very strange. At the conformal time $T=0$ the particle starts moving upwards from the $R$-axis in Figure 3 along a hyperbola in the first quadrant. As the parametric time approches $\pi-\chi_{0}$, the conformal time and radius approaches infinity. The further motion of the particle must be described in the other conformal coordinate system. At the parametric time $\pi+\chi_{0}$ the particle enters the original conformal coordinate system at infinite past and from infinitely far away, arriving at the point of departure at a parametric time $2 \pi$ corresponding to a conformal time $T=0$.

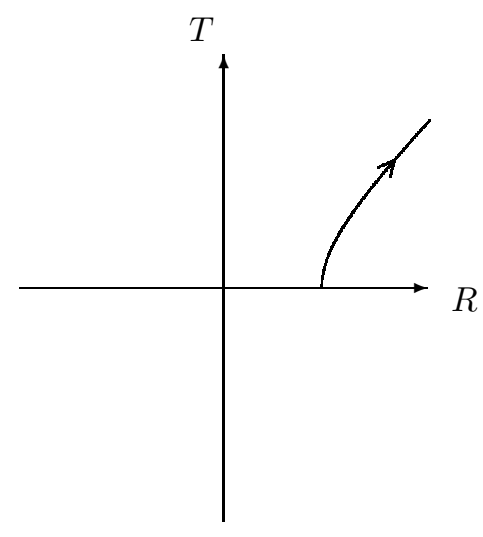

(a)

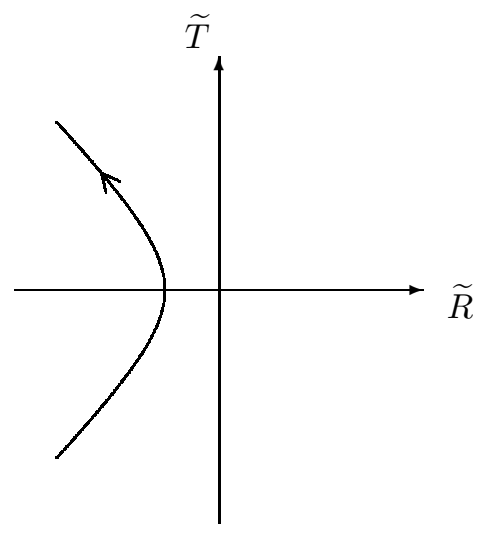

(b)

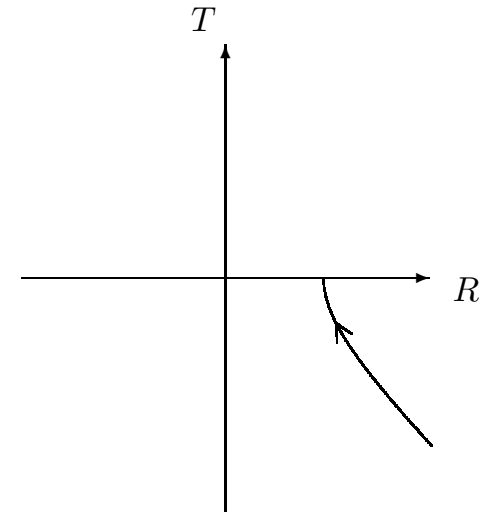

(c)

Figure 3. Minkowski diagram showing the motion of a free particle in a dust dominated universe model with positive spatial curvature, with reference to the conformal coordinate systems defined in equation (2). The world line of the particle in the $(\eta, \chi)$-system is shown in Figure 2. (a) Imagine an observer following the particle. Initially he observes clocks and meter sticks showing $T$ and $R$. There is a Big Bang at $T=0$. After the Big Bang the observer finds that $T$ and $R$ increase towards infinity. (b) The clocks and meter sticks are now replaced by new ones showing $\widetilde{T}$ and $\widetilde{R}$. They have values increasing from minus infinity. At $\widetilde{T}=0$ the expansion stops, and the universe starts contracting. Eventually $\widetilde{T}$ approaches infinity and $\widetilde{R}$ minus infinity, corresponding to a reflection through the origin. (c) Now the clocks and meter sticks are replaced by the old ones, but adjusted so that the time $T$ shown on the clocks increases from minus infinity and the distance $R$ shown on the meter sticks decreases from infinity. At the final moment, $T=0$, there is a Big Crunch. The condition that the particle shall enter the region I again is that $\chi_{0}<\pi-2 \arctan (\beta / \alpha)$.

In Figure 4 we have shown a backwards light cone of an observer at the North pole at the parametric time $\eta_{0} \in\langle\pi, 2 \pi\rangle$. $P$ is the observation event. The emission of the light is at $\eta_{A}=0, \chi_{A}=2 \pi-\eta_{0}$. At the parametric time $\left(\eta_{0}-\pi\right) / 2$ the light passes from the region I to the region II, and at time $\eta_{0}-\pi$ it passes the South pole on the sphere in Figure 2. This looks like a reflection in Figure 4. Then, at the time $\left(\eta_{0}+\pi\right) / 2$ it passes into the region I again, arriving at $P$ at $\eta=\eta_{0}$. The corresponding paths in the conformal coordinate systems are shown in Figure 5. The conformal radius of the emission point is

$$
R_{A}=-\frac{\sin \eta_{0}}{1+\cos \eta_{0}}
$$


which is positive. If the light had been emitted from $A$ in the opposite direction, it would have moved directly towards the North pole, not around the universe via the South pole. This means that $A$ would have been seen by the observer at the North pole much earlier than at $\eta_{0}$. Hence for $\pi<\eta_{0}<2 \pi$, all of the universe could have been seen, and there is no horizon. The closed universe model with radiation and dust has a horizon only for $\eta_{0}<\pi$. In a dust dominated universe the horizon exists during the expansion era, and vanishes as the universe reaches its maximum size.

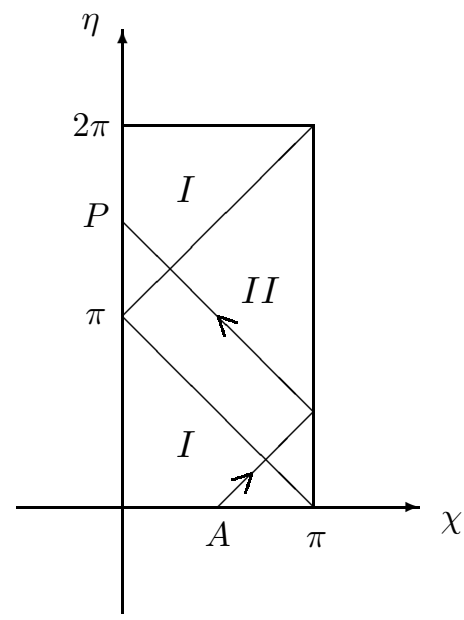

Figure 4. Past light cone of an event $P$ in the $(\eta, \chi)$-plane in a closed universe model.
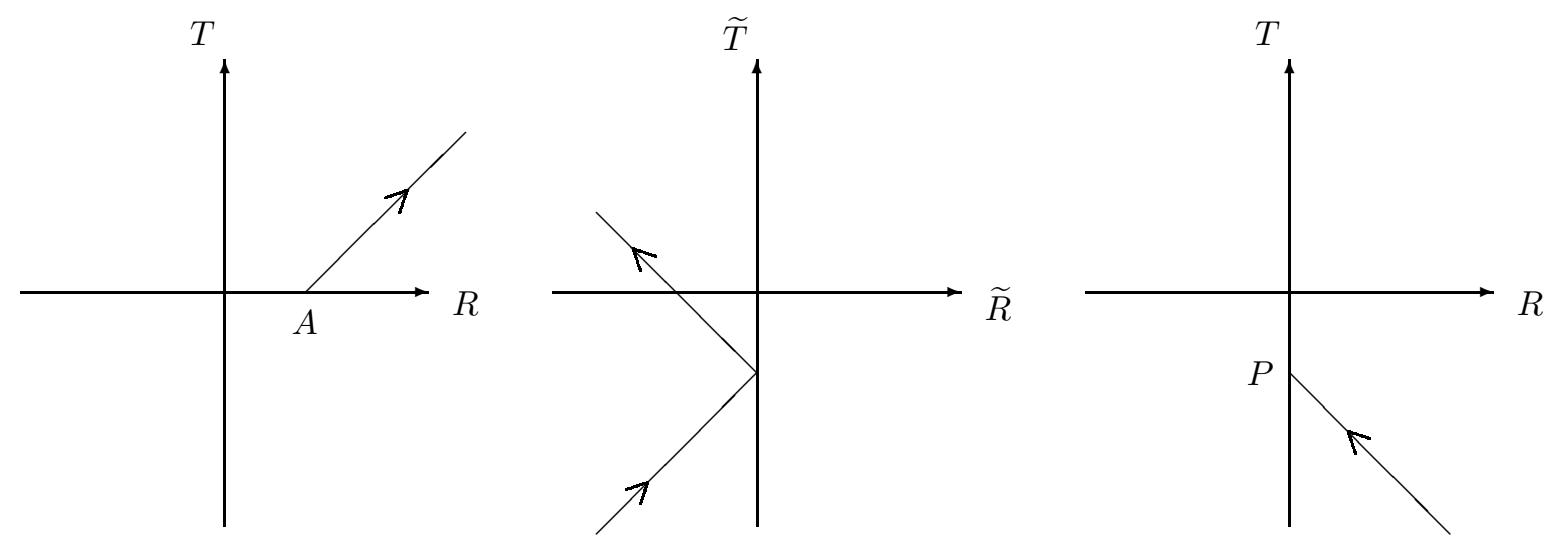

Figure 5. Past light cone of an event $P$ in the conformal coordinate systems in a closed universe model.

Again $\eta=0$ for $t=0$, and there is no continual creation. On the other hand there is continual annihilation of conformal space, as was explained by considering a similar situation in section 6 of paper [2]. In the region $\chi_{3}<\chi<\pi$ where $\chi_{3}=\pi-2 \arctan (\beta / \alpha)$, a spherical hole where space is not defined develops in the $(\widetilde{T}, \widetilde{R})$-system as shown in Figure 6 (a). It first appears at $\widetilde{R}=0$ at the point of time $\widetilde{T}=\alpha / \beta$. However, a reference particle 
with $0<\chi<\chi_{3}$ escapes the hole in the $(\widetilde{T}, \widetilde{R})$-system and enters the $(T, R)$-system as shown in Figure 6(b). All world lines end on the hyperbola given by equation (5) with $\eta_{0}=\eta_{3}$, representing the Big Crunch. Hence a person at $R=0$ will observe that the Big Crunch approaches and reaches his position at $T=-\beta / \alpha$.

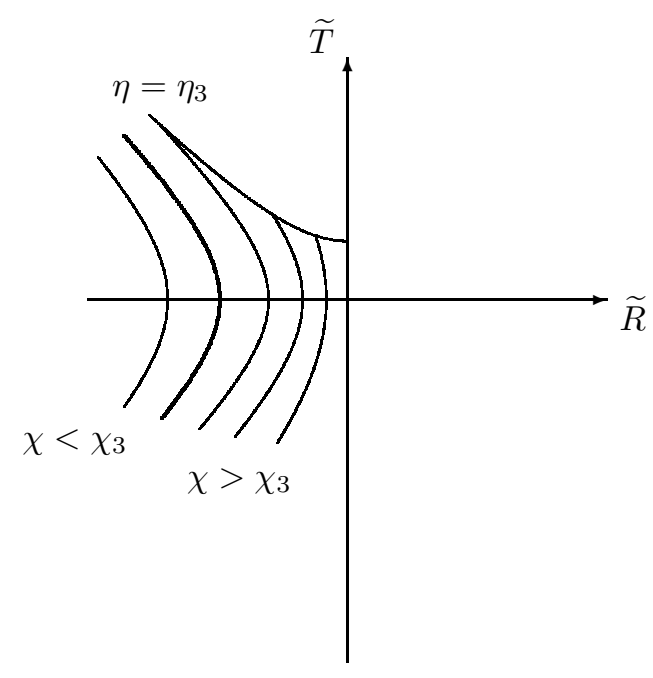

(a)

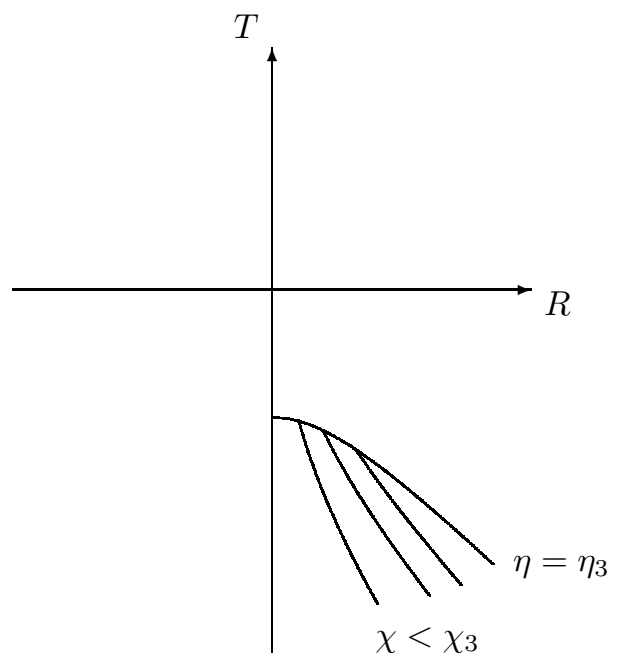

(b)

Figure 6 . The final fate of a dust and radiation filled universe with positive spatial curvature. (a) The curve $\eta=\eta_{3}$ represents the boundary of an expanding spherical hole with center at $\widetilde{R}=0$ where conformal space disappears. The figure shows world lines of particles with $\chi=$ constant. Reference particles with $\chi_{3}<\chi<\pi$ hit the hole, and those with $0<\chi<\chi_{3}$ avoid it. Note that the $\widetilde{T}$-axis corresponds to $\chi=\pi$, while the $T$-axis corresponds to $\chi=0$. (b) As $\widetilde{T}$ approaches infinity, the conformal clocks are reset to come from minus infinity, and $\widetilde{R}$ changes from negative to positive values. The conformal space then has a finite volume with decreasing size. Again, the curve $\eta=\eta_{3}$ represents the boundary where space is annihilated. The particles with $\chi=$ constant that avoided the hole in (a) come from infinitely far away and vanish when they arrive at this boundary.

\subsection{LIVE dominated universe with positive spatial curvature}

In a universe model with $k=1$ dominated by LIVE the scale factor is

$$
a(t)=\frac{1}{\widehat{H}_{\Lambda}} \cosh \left(\widehat{H}_{\Lambda} t\right)
$$

where $t$ goes from $-\infty$ to $\infty$, and $\widehat{H}_{\Lambda}$ is given in equation II:(47). This is a bouncing universe model without a Big Bang. For this model the parametric time is

$$
\eta=\arcsin \left(\tanh \left(\widehat{H}_{\Lambda} t\right)\right)
$$

where $-\pi / 2<\eta<\pi / 2$. The scale factor as expressed in parametric time is

$$
a(\eta)=\frac{1}{\widehat{H}_{\Lambda} \cos \eta} .
$$


For a LIVE dominated universe model with positive spatial curvature we shall consider three different types of conformal coordinates. The coordinates of the first type are given in equation (2). The line element then takes the form $[6,7]$

$$
d s^{2}=\frac{4}{\widehat{H}_{\Lambda}^{2}\left[1-\left(T^{2}-R^{2}\right)\right]^{2}} d s_{M}^{2} .
$$

From equations I:(17) and (20) we find the conformal scale factor

$$
A(T, R)=\frac{2 \operatorname{sgn}(R)}{\widehat{H}_{\Lambda}\left[1-\left(T^{2}-R^{2}\right)\right]} .
$$

The sign factor is due to the requirement that $A(T, R)>0$, and the fact that the denominator is positive for $T^{2}-R^{2}<1$ and negative for $T^{2}-R^{2}>1$. From Figure 7 we see that the first condition corresponds to $R>0$ and the last condition to $R<0$.

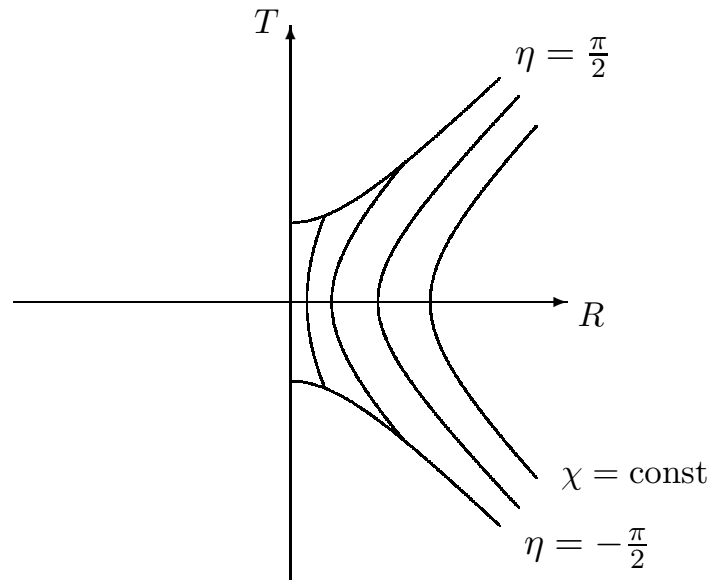

(a)

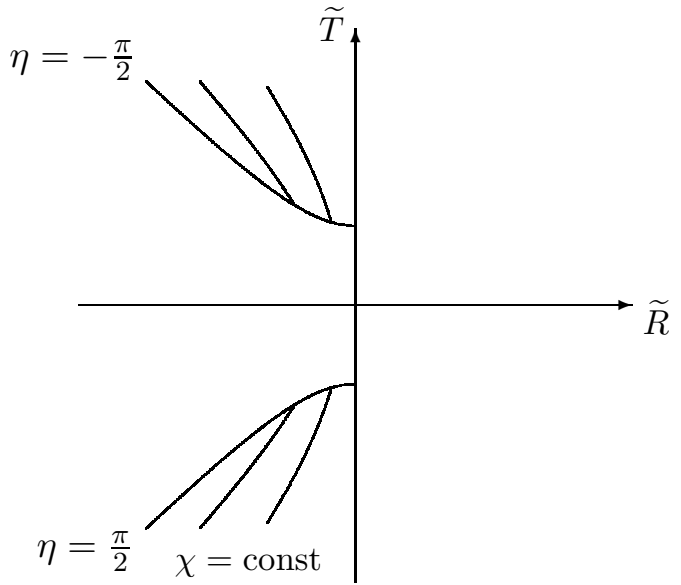

(b)

Figure 7. In the CFS space of a LIVE dominated universe with $k=1$ there is initially an empty bubble of decreasing size in which space does not exist. New space is created, and at the conformal time $T=-1$ the bubble vanishes. A particle with $\chi=\chi_{1}<\pi / 2$ comes from the boundary of the bubble in region I at a point of time $T_{1}=-\sqrt{1+R_{1}^{2}}$. It moves towards the origin, comes instantaneously to rest at $T=0$ and then accelerates outwards. As shown in part (a) of the figure a new bubble is created at $T=1$ and expands. Conformal space is annihilated at the boundary of the bubble. The particle hits the bubble at $T=\sqrt{1+R_{1}^{2}}$. A particle with $\chi=\chi_{2}$ where $\pi / 2<\chi_{2}<\pi$ comes from a different region II in spacetime, covered by the $(\widetilde{T}, \widetilde{R})$-coordinate system shown in part (b) of the figure. A bubble of conformal space appears at $\widetilde{T}=1$ and expands. The particle comes from the boundary of this bubble. It accelerates away from the origin and moves towards infinity. Then it enters the region I covered by the $(T, R)$-system. In this system it comes from spatial infinity at an infinitely past time, moves to a finite distance from the origin, and then accelerates back to spatial infinity in the infinite future. Then it enters the region II again, reappearing at infinite past in the $(\widetilde{T}, \widetilde{R})$-system from a direction opposite to the one it started its motion. Conformal space is annihilated and vanishes at $\widetilde{T}=-1$. The particle hits the boundary of space at $\widetilde{T}=-\sqrt{1+R_{2}^{2}}$. 
The parametric time $\eta$ is given in equation (18) with $-\infty<t<\infty$ corresponding to $-\pi / 2<\eta<\pi / 2$. The coordinate region I in Figure 8 defined by $-\pi / 2<\eta<\pi / 2$ and $0<\chi<\pi-|\eta|$ in the $(\eta, \chi)$-system is transformed onto the the region given by $R>0$ and $T^{2}<1+R^{2}$ in the $(T, R)$-system as shown in Figure 7a. The coordinate region II defined by $-\pi / 2<\eta<\pi / 2$ and $\pi-|\eta|<\chi<\pi$ in the $(\eta, \chi)$-system is transformed onto the region given by $\widetilde{R}<0$ and $\widetilde{T}^{2}>1+\widetilde{R}^{2}$ in the $(\widetilde{T}, \widetilde{R})$-system as shown in Figure 7b.

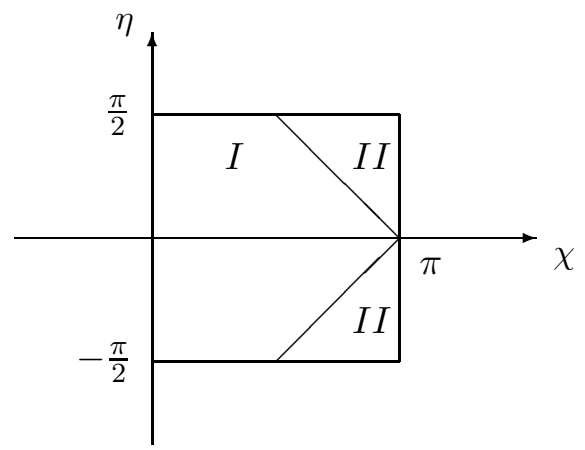

Figure 8. The $(\eta, \chi)$-coordinate regions of a LIVE dominated universe with positive curvature that are transformed into the $(T, R)$ - and $(\widetilde{T}, \widetilde{R})$-coordinate regions of Figure 7 .

We shall describe the evolution of a region where $0<\chi<\pi / 2$ in a LIVE dominated universe with positive spatial curvature. Initially there is a hole in a conformal space which is arbitrarily large in the infinitely remote past. At the boundary of the hole, the cosmic time $t$ approaches $-\infty$. The hole decreases in size with continual creation of new space and vacuum energy at the boundary. This is similar to the situation described in section 5 of paper [2]. At the conformal time $T=-1$ the hole vanishes. Then at $T=1$ the hole reappears, and there is continual annihilation. In this region every reference particle with $\chi=$ constant is created at the boundary of the first hole, and is annihilated at the boundary of the second hole. In the region with $\pi / 2<\chi<\pi$ a conformal space of finite extension, initially created at $\widetilde{T}=1$ and $\widetilde{R}=0$, is expanding with continual creation.

\subsection{New types of conformal coordinates for a LIVE dominated universe with positive spatial curvature}

V.F.Mukhanov [8] has introduced a parametric time $\widehat{\eta}=\eta-\pi / 2$ where $-\pi<\widehat{\eta}<0$. In this way he obtains the same value $\widehat{\eta}=0$ at the end of the universe for all cases $k=-1$, $k=0$ and $k=1$. However, our choice (18) is natural if one focuses on the symmetry of the function $a(t)$, i.e. on the symmetry of the expansion history of the universe.

V.F.Mukhanov uses the transformation (2) with $\eta$ replaced by $\widehat{\eta}$. Expressed by our coordinate $\eta$ this corresponds to choosing $a=\pi / 2, b=0, c=1$ and $d=0$ in equation $\mathrm{I}:(33)$. This defines a second type of conformal coordinates for LIVE dominated universe 
model with positive spatial curvature with generating function

$$
f(x)=\tan (x / 2-\pi / 4)=\frac{\tan (x / 2)-1}{\tan (x / 2)+1} .
$$

so that transformation I:(28) takes the form

$$
\widehat{T}=-\frac{\cos \eta}{\sin \eta+\cos \chi} \quad, \quad \widehat{R}=\frac{\sin \chi}{\sin \eta+\cos \chi}
$$

transforming the triangle defined by $0<\chi<\pi$ and $-\pi / 2<\eta<\chi-\pi / 2$ onto the second quadrant $\widehat{R}<0, \widehat{T}>0$, and the triangle defined by $0<\chi<\pi$ and $\chi-\pi / 2<\eta<\pi / 2$ onto the fourth quadrant $\widehat{R}>0, \widehat{T}<0$. The inverse transformation is

$$
\tan \eta=\frac{\left(\widehat{T}^{2}-\widehat{R}^{2}\right)-1}{2 \widehat{T}} \quad, \quad \cot \chi=\frac{\left(\widehat{T}^{2}-\widehat{R}^{2}\right)+1}{2 \widehat{R}} .
$$

One may wonder if it is possible to define a new type of conformal coordinates by choosing $a=-\pi / 2, b=0, c=1$ and $d=0$ in equation I:(33). Then the generating function would be

$$
f(x)=\tan (x / 2+\pi / 4)=\frac{1+\tan (x / 2)}{1-\tan (x / 2)} .
$$

so that the transformation I:(28) would take the form

$$
\widetilde{T}=\frac{\cos \eta}{\cos \chi-\sin \eta} \quad, \quad \widetilde{R}=\frac{\sin \chi}{\cos \chi-\sin \eta}
$$

Replacing $\chi$ by $\pi-\chi$ and $R$ by $-R$, this transformation has the same form as in (2). Changing the sign of $R$ corresponds to replacing the coordinate $\theta$ by $\pi-\theta$ and $\phi$ by $\phi+\pi$. This is an antipodal transformation, i.e. a reflection through the origin in $S^{3}$. Hence nothing new is obtained physically. The CFS coordinate $\widehat{T}$ has the value zero at the beginning and the end of the universe. Again we see that the CFS time coordinate behaves strangely. In the limit that the CFS time approaches infinity, it is reset to come from minus infinity as shown in Figure 9.

In order to obtain a correct physical interpretation of this figure, one must take account of the difference between coordinate distances and physical distances in the radial direction, $d l=A(R, T) d R$. From equations (19), I:(29) and (23) the scale factor takes the form

$$
A(T, R)=-\frac{\operatorname{sgn} R}{\widehat{H}_{\Lambda} T} .
$$

where we have omitted the hat and the tilde on $T$ and $R$.

Looking at Figure 9, we have a coordinate description of particles with constant $\chi$ in the $(\widehat{T}, \widehat{R})$-system.

It may be noted that the transformation equation (23) can also be used for universe models with $k=1$ containing dust and radiation, but with different coordinate domains. 


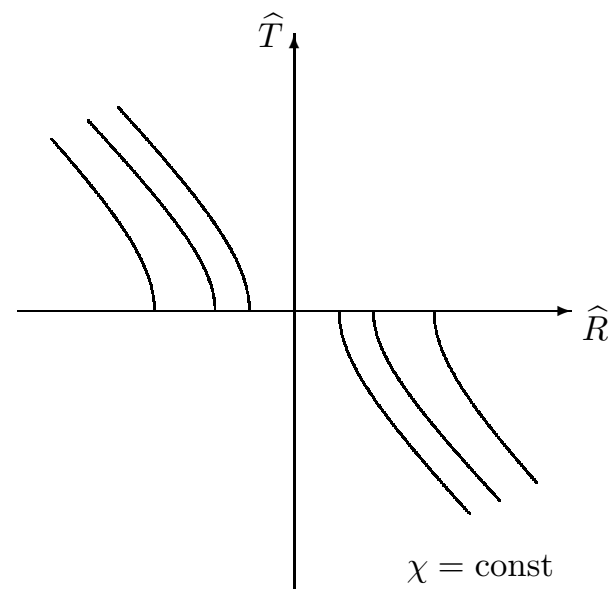

Figure 9. World lines of reference particles with $\chi=$ constant (defining the Hubble flow) in a LIVE dominated universe as observed from the CFS system defined by the transformation (23).

\section{Penrose diagrams}

By applying the rule in Appendix B of paper [1] for composing generating functions one may obtain a deeper understanding of the conformal coordinate transformations and find new ones. Let us first consider the transformations to CFS coordinates of type III in universe models with negative spatial curvature. Here the generating function in II:(67) is obtained as a composition of the generating functions in II:(55) and II:(71).

Combining the generating functions (11) and II:(71) we obtain the generating function

$$
f(x)=-\cot (x / 2) .
$$

and the transformation

$$
T=\frac{\sin \eta}{\cos \eta-\cos \chi} \quad, \quad R=\frac{\sin \chi}{\cos \chi-\cos \eta},
$$

In the same way as in section 2.4 this only represents a composition of the antipodal transformation with the transformation (1), and therefore nothing new is obtained physically.

We have seen in section 3 of paper [1] that it is possible to transform away spatial curvature. In the same way we obtain a more general transformation from spaces with curvature $k_{1}$ to spaces with curvature $k_{2}$ if the generating function $f$ satisfies the relation

$$
f^{\prime}(u) f^{\prime}(v) S_{k_{1}}\left(\frac{u-v}{2}\right)^{2}=S_{k_{2}}\left(\frac{f(u)-f(v)}{2}\right)^{2} .
$$

Composition of generating functions can also be used to find coordinates used in Penrose diagrams, as we will investigate below. Our formalism can be utilized to give simple 
deductions of the transformations that lead to the Penrose diagrams. Then equation I:(29) for the CFS scale factor is modified to give a corresponding conformal Einstein space (CES) scale factor, given as

$$
A(T, R)=\frac{a(\eta(T, R)) S_{k}(\chi(T, R))}{|\sin R|} .
$$

By means of the composition rule in Appendix B of paper [1] we shall first find a transformation from spaces with negative curvature to spaces with positive curvature, corresponding to Einstein's static universe.

By means of a composition of the generating function in II:(10) and the inverse of the generating function in I:(28), we obtain the generating function

$$
f(x)=-\frac{1}{T_{i}} e^{-x} .
$$

This represents a transformation from a space with $k=-1$ and CFS coordinates of type I to a space with $k=1$ and CFS coordinates of type I. Equation I:(28) then implies that the transformed coordinates $T$ and $R$ are given by

$$
\tan T=\frac{2 T_{i} e^{\eta} \cosh \chi}{1-T_{i}^{2} e^{2 \eta}} \quad, \quad \tan R=\frac{2 T_{i} e^{\eta} \sinh \chi}{1+T_{i}^{2} e^{2 \eta}} .
$$

The inverse transformation is

$$
T_{i} e^{\eta}=\sqrt{\frac{\cos R-\cos T}{\cos R+\cos T}} \quad, \quad \tanh \chi=\frac{\sin R}{\sin T} .
$$

Using equation (34) and the transformation I:(17) we find that the line element I:(1) with $k=-1$ as expressed in CES coordinates takes the form

$$
d s^{2}=\frac{a(\eta(T, R))^{2}}{\cos ^{2} R-\cos ^{2} T}\left(-d T^{2}+d R^{2}+\sin ^{2} R d \Omega^{2}\right)=\frac{a(\eta(T, R))^{2}}{\cos ^{2} R-\cos ^{2} T} d s_{E}^{2} .
$$

where $d s_{E}^{2}$ is the line element of Einstein's static universe.

In the case of the Milne universe the transformation to the CES coordinates reduces to

$$
\tan T=\frac{t \cosh \chi}{1-t^{2}} \quad, \quad \tan R=\frac{t \sinh \chi}{1+t^{2}} .
$$

transforming the first quadrant $t>0$ and $\chi>0$ onto the triangle $0<R<\pi / 2$ and $R<T<\pi-R$, with inverse transformation

$$
t=\sqrt{\frac{\cos R-\cos T}{\cos R+\cos T}} \quad, \quad \tanh \chi=\frac{\sin R}{\sin T} .
$$

The CES form of the line element for the Milne universe is

$$
d s^{2}=\frac{1}{(\cos R+\cos T)^{2}} d s_{E}^{2} .
$$

Taking a composition of the generating function in II:(55) and the inverse of the generating function in (1), we obtain the generating function

$$
f(x)=2 \arctan (\tanh (x / 2)) .
$$


This represents a transformation from a space with $k=-1$ and CFS coordinates of type II to a space with $k=1$ and CFS coordinates of type I. Equation I:(28) then implies that the transformed coordinates $T$ and $R$ are given by

$$
\tan T=\frac{\sinh \eta}{\cosh \chi} \quad, \quad \tan R=\frac{\sinh \chi}{\cosh \eta} .
$$

If one transforms from a universe model with dust and radiation which begins at $\eta=0$, this transformation maps the first quadrant $\chi>0, \eta>0$ onto the triangle $0<\widehat{T}<\pi / 2$ and $0<\widehat{R}<\pi / 2-\widehat{T}$. On the other hand, if one transforms from a universe which is vacuum dominated and infinitely old, the fourth quadrant $\chi>0, \eta<0$ is mapped onto the triangle $-\pi / 2<\widehat{T}<0$ and $0<\widehat{R}<\pi / 2+\widehat{T}$. The inverse transformation is

$$
\tanh \eta=\frac{\sin T}{\cos R} \quad, \quad \tanh \chi=\frac{\sin R}{\cos T} .
$$

Using equation (31) and the transformation (41) we find that the line element I:(17) for a universe model with $k=-1$ as expressed in CES coordinates takes the form

$$
d s^{2}=\frac{a(\eta(T, R))^{2}}{\cos ^{2} T-\sin ^{2} R}\left(-d T^{2}+d R^{2}+\sin ^{2} R d \Omega^{2}\right)=\frac{a(\eta(T, R))^{2}}{\cos ^{2} T-\sin ^{2} R} d s_{E}^{2} .
$$

We shall next find a transformation from flat spaces to spaces with positive curvature, corresponding to Einstein's static universe. Taking a composition of the generating function $f(x)=x$ for the first type of CFS coordinates in flat space, and the inverse of the generating function in (11), we obtain the generating function

$$
f(x)=2 \arctan (x),
$$

which is the inverse of the generating function given in formula (11). Hence the coordinate transformation is the inverse of the one given in equation (2),

$$
\cot T=\frac{1-\left(\eta^{2}-\chi^{2}\right)}{2 \eta} \quad, \quad \cot R=\frac{1+\left(\eta^{2}-\chi^{2}\right)}{2 \chi} .
$$

with inverse transformation

$$
\eta=\frac{\sin T}{\cos T+\cos R} \quad, \quad \chi=\frac{\sin R}{\cos T+\cos R}
$$

Again using (31), this time in combination with the transformation (45), we find the line element for flat space in CES coordinates

$$
d s^{2}=\frac{a(\eta(T, R))^{2}}{(\cos T+\cos R)^{2}}\left(-d T^{2}+d R^{2}+\sin ^{2} R d \Omega^{2}\right)=\frac{a(\eta(T, R))^{2}}{(\cos T+\cos R)^{2}} d s_{E}^{2} .
$$

Note that the line element (38) can be immediately obtained by putting $a=1$ in equation (46). This is a consequence of the fact that the Milne universe is a coordinate transformation of the interior of the future light cone of the point $(0,0)$ in the static and spatially flat Minkowski spacetime. 
As a simple example we shall find the CES coordinates for the Minkowski spacetime with line element

$$
d s^{2}=-d t^{2}+d r^{2}+r^{2} d \Omega^{2} .
$$

From equation (31) and the transformation (45) it follows that the CES scale factor for the Minkowski spacetime is [9]

$$
A(T, R)=\frac{1}{\cos T+\cos R}
$$

It may be noted that in the case of universe models with positive spatial curvature, $\eta$ and $\chi$ are CES coordinates. For a de Sitter universe with $k=1$ one obtains by using equation (19), the CES form of the line element

$$
d s^{2}=\frac{1}{\widehat{H}_{\Lambda}^{2} \cos ^{2} \eta} d s_{E}^{2},
$$

correcting an error in reference [10].

\section{Some physical properties of universe models using CFS coordinates}

The parametric time $\eta$ is widely used in cosmology because many formulae become simpler using this than when expressed in termes of cosmic time $t$. Important examples are the formulae I:(9) and I:(10) for the radius of the particle horizon and the expressions II:(30), II:(31) and (11), (12) for the cosmic scale factor in a curved universe with radiation and dust.

One may wonder if there are any similar advantages in using the CFS coordinates $T$ and $R$. According to G.Endean [5, 11-12] there are several. He argues that the picture of the universe obtained with reference to the CFS coordinates is more correct and relevant when interpreting observational data than the usual picture referring to cosmic time $t$ (or the parametric time $\eta$ ) and the standard radial coordinate $\chi$. The criterion for a Big Bang is that $A(0, R)=0$. This will be the case if $a$ converges to 0 faster than $B$ when $T \rightarrow 0$.

Endean claims for example that there was no Big Bang because the recession velocity vanishes at $t=0$ (see equations II:(60) and II:(75)). The recession velocity is the velocity of a galaxy with $\chi=$ constant relative to the CFS system. We prefer the following interpretation: The mentioned expressions tell that the reference particles of the CFS system are initially at rest relative to the galaxes. Then the CFS particles accelerate away from the galaxes, which in no way implies that there was no Big Bang.

Endean also claims that in a dust dominated universe with $k=1$ there will not be a Big Crunch. The reason for this is the following. At $\chi=0$ the coordinate transformation (2) gives

$$
T=\tan \frac{\eta}{2}
$$

Furthermore, the cosmic scale factor $a=\alpha(1-\cos \eta)$ has a maximum value $a_{\max }=2 \alpha$ for $\eta=\pi$. This corresponds to a finite cosmic time $t_{1}=\pi \alpha$, but to an infinite CFS time. Hence, in the CFS picture the closed dust dominated universe expands for an infinitely long time, and a Big Crunch never happens. 
However, the CFS clocks are not standard clocks like the cosmic clocks showing $t$. They are coordinate clocks going at the rate that is adjusted to be in accordance with the first of equations (2). Compared to the standard clocks and clocks showing parametric time, the CFS clocks go increasingly fast. Hence the non-vanishing of a Big Crunch in the CFS picture is a coordinate effect. Further criticism of Endean's interpretation of the CFS picture has been given by L.Querella [13].

Let us consider a few examples in which the conformal time at $\chi=0$ can be very simply related to the cosmic time, namely radiation dominated universes with negative, vanishing and positive spatial curvature. With reference to the CFS systems given respectively in the transformations II:(49), II:(68) and (2) we find for clocks at $\chi=0$,

$$
T=\frac{t}{a} .
$$

In a radiation dominated universe $[14,15]$

$$
a=\sqrt{2 \beta t-k t^{2}} .
$$

Hence

$$
T=\sqrt{\frac{t}{2 \beta-k t}} .
$$

\subsection{The CFS Hubble parameter of some universe models}

The behaviour of the conformal space in different universe models may be investigated by calculating the CFS Hubble parameter defined in equation I:(48). We first consider negatively curved universe models with respectively dust and radiation as described in CFS systems of type I. Using equation II:(35) we obtain

$$
H_{R}=\frac{2 T_{i} T}{\left(\sqrt{T^{2}-R^{2}}-T_{i}\right)^{3}}
$$

for a dust dominated universe, and by means of equation II:(36) we get

$$
H_{R}=\frac{2 T_{i}^{2} T}{\left(T^{2}-R^{2}-T_{i}^{2}\right)^{2}}
$$

for a radiation dominated universe. As shown in section 5 of paper [2] there is continual creation at the boundary of the conformal space given by $T^{2}-R^{2}=T_{i}^{2}$. Hence the CFS Hubble parameter for both of these universe models approaches infinity at the boundary with continual creation.

The CFS Hubble parameter for a negatively curved radiation dominated universe with conformal coordinates of type II and III is

$$
H_{R}=\operatorname{sgn}(T) \frac{\left(1+\widehat{T}^{2}-\widehat{R}^{2}\right)^{2}-4 \widehat{T}^{2}\left(\widehat{T}^{2}-\widehat{R}^{2}\right)}{4 \beta \widehat{T}^{2}}
$$

Correspondingly for a flat universe one obtains

$$
H_{R}=\frac{\left|T^{2}-R^{2}\right|\left(3 T^{2}+R^{2}\right)}{\beta T^{2}}
$$


For a universe with positive spatial curvature it is

$$
H_{R}=\operatorname{sgn}(T) \frac{\left[1-\left(\widehat{T}^{2}-\widehat{R}^{2}\right)\right]^{2}-4 \widehat{T}^{2}\left(\widehat{T}^{2}-\widehat{R}^{2}\right)}{4 \beta \widehat{T}^{2}}
$$

The Hubble parameter for a LIVE dominated universe model with $k=-1$ and CFS coordinates of type I is

$$
H_{R}=\sqrt{\Omega_{0}} \widehat{H}_{0} \frac{T}{T_{f}}
$$

In this case the conformal Hubble parameter in independent of the position $R$. The Hubble parameter for the same universe model but with CFS coordinates of type II and III is

$$
H_{R}=-\widehat{H}_{\Lambda} \operatorname{sgn}(T)
$$

where we have omitted the hat and the tilde on $T$. This formula is also valid for a flat LIVE dominated universe. The conformal space therefore expands for $T<0$ and contracts for $T>0$. This behaviour is a result of two competing motions. The Hubble flow expands exponentially. But as seen from Figure 12 in paper [2], the conformal reference frame contracts relative to the Hubble flow. The sign of conformal Hubble parameter shows that the expansion dominates for $T<0$ corresponding to the region $-\chi<\eta<0$ in the $(\eta, \chi)$-plane, while the contaction of the conformal system dominates for $T>0$ corresponding to the region $0<\chi<-\eta$.

From equations I:(48) and (21) it follows that the CFS Hubble parameter for a LIVE dominated universe model with positive spatial curvature in CFS coordinates of type I is

$$
H_{R}=\operatorname{sgn}(R) \widehat{H}_{\Lambda} T
$$

The corresponding Hubble parameter in CFS coordinates of type II and III is

$$
H_{R}=\operatorname{sgn}(R) \widehat{H}_{\Lambda}
$$

\subsection{The CFS age of some universe models}

We shall now calculate the conformal age of some universe models containing radiation only. They are of course not realistic universe models, but we shall calculate their conformal age as an illustration.

Consider first a negatively curved universe model. The parametric age $\eta_{0}$ is determined from the normalization $a\left(\eta_{0}\right)=1$ in equation II:(30), which gives

$$
\sinh \eta_{0}=1 / \beta .
$$

The cosmic age is given in equation II:(31) with $\alpha=0$. Together with equation (63) this leads to

$$
t_{0}=\sqrt{1+\beta^{2}}-\beta,
$$

where $\beta$ is given in equation II:(32) and has the value $9.1 \cdot 10^{-3}$ for a radiation density equal to that of the background radiation at the present time. 
We shall now calculate the present value of the time $T_{0}$ in a CFS system of type I. It is given by equation II:(12) which shows that $T_{0}$ is $R$-dependent and given by

$$
T_{0}=\sqrt{R^{2}+(1 / 4)\left(1+\sqrt{1+\beta^{2}}\right)^{2}},
$$

The initial time of the universe model is

$$
T_{i}=\sqrt{R^{2}+(1 / 4) \beta^{2}} .
$$

According to equation I:(5) the unit of time is

$$
l_{0} / c=\left(H_{0} \sqrt{1-\Omega_{\gamma 0}}\right)^{-1}=13.9 \cdot 10^{9} y,
$$

where we have used the value of $H_{0}$ in reference [16], and that $\Omega_{\gamma 0}=6 \cdot 10^{-5}$. At $R=0$ the dimensional initial time is

$$
\left(l_{0} / c\right) T_{i}=\frac{l_{0} \beta}{2 c}=63 \cdot 10^{6} y .
$$

The CFS age of the universe is $T_{0}-T_{i}$, where $T_{0}$ and $T_{i}$ are given in equations (65) and (66). Hence the conformal age at $R=0$ of this radiation dominated universe model is

$$
\frac{l_{0}}{c}\left(T_{0}-T_{i}\right)=\frac{l_{0}}{2 c}\left\{\left(1+\sqrt{1+\beta^{2}}\right)-\beta\right\}=13.8 \cdot 10^{9} y .
$$

Using equations (65) and II:(31) we find that the conformal age and the cosmic age at $R=0$ are related by

$$
T_{0}-T_{i}=\frac{1}{2}\left(1+t_{0}\right)
$$

Consider next a negatively curved universe model with CFS coordinates of type II. The CFS age of this universe is

$$
\widehat{T}_{0}=\sqrt{1+\beta^{2}}-\sqrt{\widehat{R}^{2}+\beta^{2}}
$$

where $0<\widehat{T}_{0}<1$ and $0<\widehat{R}<1-\widehat{T}_{0}$. The conformal age and the cosmic age at $\widehat{R}=0$ are related by

$$
\widehat{T}_{0}=\left\{\begin{array}{ll}
t_{0} & \text { for } \quad 0<t_{0}<1 \\
\frac{1}{t_{0}} & \text { for } t_{0}>1
\end{array} .\right.
$$

We then consider a flat, radiation dominated universe. The cosmic age of this universe is

$$
t_{0}=\frac{1}{2 \beta}
$$

where $\beta$ is given in equation II:(77). As described in a CFS coordinate system given in equation II:(72), there are some strange coordinate effects. In the CFS coordinate system the universe seems to be divided into two parts. The part $U_{1}$ outside the future light cone of $(\eta, \chi)=(0,0)$ appears in the conformal coordinate system with a positive time, coming from $\widehat{T}=0$ and approaching infinity. The part $U_{2}$ inside the light cone appears with a 
negative conformal time, coming from minus infinity and converging towards $(\widehat{T}, \widehat{R})=$ $(0,0)$.

The conformal age of $U_{1}$ is

$$
T_{0}=\frac{1}{2}\left(\sqrt{R^{2}+\beta^{2}}-\beta\right) .
$$

For the other part $U_{2}$ one can only define the time left until a Big Crunch of the conformal space, which is given by

$$
T_{f}-T_{0}=\frac{1}{2}\left(\sqrt{R^{2}+\beta^{2}}+\beta\right)-R
$$

We finally consider a positively curved, radiation dominated universe. Its cosmic age is

$$
t_{0}=\beta-\sqrt{\beta^{2}-1},
$$

where $\beta$ is given in equation (13). The radiation dominated universe is represented by the region $\eta<\pi$ in Figure 2. This region has two parts I and II. In part I the conformal time is positive, and the age is

$$
T_{0}=\sqrt{R^{2}+\beta^{2}}-\sqrt{\beta^{2}-1} .
$$

On the other hand, in part II the conformal time is negative, and the conformal space disappears at $\widetilde{T}=0$. In this case the conformal time left until the space disappears is

$$
-T_{0}=\sqrt{R^{2}+\beta^{2}}+\sqrt{\beta^{2}-1} .
$$

\subsection{Recession velocity and cosmic redshift in CFS space}

We have considered several types of conformally flat spacetime coordinate systems. One series has $a=b=d=0$ in equation I:(33). Then the generating function reduces to

$$
f(x)=c / I_{k}(x / 2),
$$

and the general expression I:(46) for the recession velocity takes the form

$$
V=\frac{2 k R T}{c^{2}+k\left(R^{2}+T^{2}\right)} .
$$

The corresponding formula for the Doppler shift factor given in equation I:(50) is

$$
D(T, R)=\left\{\frac{c^{2}+k(T+R)^{2}}{c^{2}+k(T-R)^{2}}\right\}^{1 / 2} .
$$

For $k=0$ equation I:(50) gives

$$
D(T, R)=\frac{b(T+R-d)-c}{b(T-R-d)-c} .
$$


We shall now find an expression for the redshift $z$ of an object at $R$ emitting light at a time $T$ as observed by an observer at $R=0$ at a time $T_{0}$. Since light has a contant velocity 1 in the CFS coordinates, this means that $R=T_{0}-T$. In the case of the Einstein de Sitter universe, i.e. a flat dust dominated universe, the constants in equation (82) have the values $b=2, c=2$ and $d=-1$, giving

$$
D(T, R)=\frac{T_{0}}{2 T-T_{0}} .
$$

Using this together with equation I:(51) we find for the CFS redshift $z$,

$$
1+z=\left(\frac{2 T-T_{0}}{T}\right)^{2}
$$

The corresponding expression for the redshift i a flat LIVE dominated universe, i.e. the de Sitter universe is

$$
1+z=\frac{T}{2 T-T_{0}}
$$

Using equation I:(51) we find that the cosmic redshift $z$ in a flat universe with $f(x)=$ $-1 / x$ is given by

$$
1+z=\frac{T+R}{T-R} \frac{A\left(T_{0}, 0\right)}{A(T, R)} \quad, \quad R=T_{0}-T
$$

where $T$ is the emission time and $T_{0}$ the time of observation.

The redshift $z$ in a universe with $k=-1, f(x)=\tanh (x / 2)$

$$
1+z=\sqrt{\frac{1-(T+R)^{2}}{1-(T-R)^{2}}} \frac{A\left(T_{0}, 0\right)}{A(T, R)} .
$$

and in a universe with $k=1, f(x)=\tan (x / 2)$ are given by the expression

$$
1+z=\sqrt{\frac{1+(T+R)^{2}}{1+(T-R)^{2}}} \frac{A\left(T_{0}, 0\right)}{A(T, R)} .
$$

\section{Conclusion}

FRW universe models with positive spatial curvature have been described in CFS coordinates. As in models with negative and vanishing curvature, described in paper II in this series, the motion of the free particles defining the Hubble flow with cosmic time, is utterly strange in these coordinates.

We have seen that in order to describe a univers model with positive spatial curvature using CFS coordinates, the universe must be covered by two coordinate domains, which we have called I and II in Figure 2. Let us first describe the evolution of the CFS space in the domain I. Physically the FRW universe with radiation and dust and with positive curvature comes from a Big Bang singularity with vanishing spatial extension. The corresponding coordinate range of the coordinate $\chi$ which is comoving with the Hubble flow is $\langle 0, \pi\rangle$ at an arbitrary time. The CFS coordinate space appears as a Big Bang 
happening everywhere at $T=0$, and the universe expands. In the infinitely far future a new CFS coordinate system $(\widetilde{T}, \widetilde{R})$ must be introduced, and the clocks are reset from plus to minus infinity. In this coordinate system the CFS space first contracts with a decreasing velocity which stops at $\widetilde{T}=0$, and then expands. Hence the evolution of the CFS coordinate space gives the impression of a space that accelerates outwards. This is however an illusion due to the motion of the CFS reference frame.

Thereafter a spherical hole in which space, dust and radiation vanish, appears at $\widetilde{R}=0$ at the point of time

$$
\widetilde{T} \cdot \frac{l_{0}}{c}=\frac{\alpha}{\beta} \cdot \frac{l_{0}}{c}=\frac{\Omega_{m 0}}{2 \sqrt{\Omega_{\gamma 0}}\left(\Omega_{0}-1\right) H_{0}},
$$

where we have reintroduced the time unit $l_{0} / c=H_{0}^{-1}\left(\Omega_{0}-1\right)^{-1 / 2}$ defined in section 2 of reference [1]. This corresponds to a parametric time where the FRW space contracts. The CFS bubble expands with superluminal velocity as illustrated in Figure 6 . In this case there is continual annihilation of space, matter and energy. Particles with $\chi>\chi_{3}$ hit the bubble and vanish, while particles with $\chi<\chi_{3}$ avoid the bubble. They disappear at an infinitely far future, only to reappear at an infinitely remote past, now approaching the observer.

This bubble does not appear if there is only dust or only radiation in the universe. In a universe with dust only, $\beta=0$, we have that $\chi_{3}=\pi$. The FRW universe only exists for $0 \leq \chi \leq \pi$. Hence if $\chi_{3}=\pi$, there is no Hubble observer hitting the bubble. This means that no bubble appears. In a universe with positive spatial curvature containing dust only the universe ends at a parametric time $\eta_{3}=2 \pi$. In this case the hyperbola $\eta=\eta_{3}$ in Figure 6, representing the surface of the bubble in which space vanishes, is replaced by the negative $\widetilde{R}$-axis and the positive $R$-axis. This means that the whole CFS space suddenly vanishes at the point of time $T=0$, corresponding to a Big Crunch. A universe with radiation only will evolve in a similar manner allthough $\eta_{3}=\pi$ in this case. Hence the continual annihilation of the CFS space in a universe with dust and radiation is replaced by a Big Crunch in universes with dust or radiation only.

In a LIVE dominated universe there is a similar behaviour. Considering first the region $0<\chi<\pi / 2$ there is initially a hole which is arbitrarily large in the infinitely remote past. It decreases in size and vanishes at a point of time $T=-1$. Hence for $T<-1$ there is continual creation of CFS space and vacuum energy at the boundary of the hole. At $T=1$ the hole reappears. It expands, and for $T>1$ there is continual annihilation of space and energy. In the region $\pi / 2<\chi<\pi$ the behaviour is more complicated. A bubble of conformal space appears at $\widetilde{T}=1$ and expands. In the limit as $\widetilde{T}$ approaches infinity this region of the universe evolves from the region II into the region I in Figur 7. The clocks are then reset to come from minus infinity, and the conformal radial coordinate changes from negative to positive values. The CFS coordinate space of the universe now contracts, but it accelerates outwards so that the contraction is replaced by an expansion at $T=0$. Then, as $T$ approaches infinity, the region $\pi / 2<\chi<\pi$ of the universe evolves from the region I into region II. Finally this region of the universe contracts and is continually annihilated, vanishing at $\widetilde{T}=-1$.

In part II of this series we have considered transformations from a description of FRW universe models with negative and vanishing spatial curvature as described with cosmic 
time and standard radial coordinates to a description in terms of CFS coordinates. In section 3 of the present paper we have shown that by composing these transformations with the inverse of transformations described in section 2 we obtain a form of the line element with a scale factor times Einstein's static universe which has positive spatial curvature. In this way we obtain Penrose diagrams where infinitely remote regions in spacetime is mapped onto a finite region.

Finally we have deduced formulae for some physical properties of the FRW universe models using CFS coordinates. In particular we have found formulae for the CFS Hubble parameter, the age, the recession velocity and the cosmic redshift of some universe models.

Maybe the most important difference between the standard coordinates and the CFS coordinates is that the standard coordinates are comoving with a cosmic reference frame consisting of free particles defining the Hubble flow, while the CFS coordinates are comoving with an accelerated reference frame. The reference particles of the cosmic frame have a recession velocity $V$ relative to the CFS system. This implies that the CFS simultaneity is different from the cosmic simultaneity. Therefore the CFS space is different from the cosmic space.

We have shown in the present series of papers that this difference makes the CFS description of the universe radically different from the description in terms of cosmic time and space. For instance, in some universe models the Big Bang and the Big Crunch in the FRW description are replaced by continual creation and annihilation. Both descriptions are in accordance with the observations of our universe and concern the same reality, but they refer to time coordinates with different simultaneities.

\section{References}

1. Ø.Grøn and S.Johannesen, FRW Universe Models in Conformally Flat Spacetime Coordinates. I: General Formalism, Eur.Phys.J.Plus 126, 28 (2011).

2. Ø.Grøn and S.Johannesen, FRW Universe Models in Conformally Flat Spacetime Coordinates. II: Universe models with negative and vanishing spatial curvature, Eur.Phys.J.Plus 126, 29 (2011).

3. G.U.Varieschi, A Kinematical Approach to Conformal Cosmology, Gen.Rel.Grav. 42, 929 - 974 (2010).

4. M.Ibison, On the conformal forms of the Robertson-Walker metric, J.Math.Phys. 48, 122501-1 - 122501-23 (2007).

5. G.Endean, Cosmology in Conformally Flat Spacetime, The Astrophysical Journal 479, 40 - 45 (1997).

6. E.Eriksen and Ø.Grøn, The De Sitter Universe Models, Int.J.Mod.Phys. D4, 115 159 (1995).

7. A.Lasenby and C.Doran, Closed Universes, de Sitter Space and inflation. Phys.Rev. D71, 063502 (2005). 
8. V.F.Mukhanov, Physical foundations of cosmology, Cambridge University Press, (2005).

9. S.M.Carroll, Spacetime and Geometry, Addison Wesley, (2004), Appendix H.

10. S.W.Hawking and G.F.R.Ellis, The large scale structure of space-time, Cambridge University Press, (1973), equation (5.8).

11. G.Endean, Redshift and the Hubble Constant in Conformally Flat Spacetime, The Astrophysical Journal 434, 397 - 401 (1994).

12. G.Endean, Resolution of Cosmological age and redshift-distance difficulties, Mon.Not. R.Astron.Soc. 277, 627 - 629 (1995).

13. L.Querella, Kinematic Cosmology in Conformally Flat Spacetime, The Astrophysical Journal 508, 129 - 131 (1998).

14. Ø.Grøn and S.Hervik, Einstein's General Theory of Relativity, Springer, (2007), ch.11.

15. Ø.Grøn, Lecture Notes on the General Theory of Relativity, Springer, 2009, p 208.

16. S.H.Suyu et.al., Dissecting the gravitational lens B1608+656. II. Precision measurements of the Hubble constant, spatial curvature, and the dark energy energy equation of state, arXiv:0910.2773. 